OPEN ACCESS

Edited by:

Thomas Deller,

Goethe-University, Germany

Reviewed by:

Monica Diluca,

University of Milan, Italy

Claus Pietrwik,

University of Mainz, Germany

Jochen Walter,

University of Bonn, Germany

${ }^{*}$ Correspondence:

Paul Saftig

psaftig@biochem.uni-kiel.de

Received: 31 October 2016 Accepted: 05 December 2016 Published: 21 December 2016

Citation:

Seipold $L$ and Saftig P (2016) The Emerging Role of Tetraspanins in the Proteolytic Processing of the Amyloid

Precursor Protein

Front. Mol. Neurosci. 9:149.

doi: 10.3389/fnmol.2016.00149

\section{The Emerging Role of Tetraspanins in the Proteolytic Processing of the Amyloid Precursor Protein}

\author{
Lisa Seipold and Paul Saftig * \\ Institut für Biochemie, Christian-Albrechts-Universität zu Kiel (CAU), Kiel, Germany
}

Tetraspanins are a family of ubiquitously expressed and conserved proteins, which are characterized by four transmembrane domains and the formation of a short and a large extracellular loop (LEL). Through interaction with other tetraspanins and transmembrane proteins such as growth factors, receptors and integrins, tetraspanins build a wide ranging and membrane spanning protein network. Such tetraspanin-enriched microdomains (TEMs) contribute to the formation and stability of functional signaling complexes involved in cell activation, adhesion, motility, differentiation, and malignancy. There is increasing evidence showing that the tetraspanins also regulate the proteolysis of the amyloid precursor protein (APP) by physically interacting with the APP secretases. CD9, CD63, CD81, Tspan12, Tspan15 are among the tetraspanins involved in the intracellular transport and in the stabilization of the gamma secretase complex or ADAM10 as the major APP alpha secretase. They also directly regulate, most likely in concert with other tetraspanins, the proteolytic function of these membrane embedded enzymes. Despite the knowledge about the interaction of tetraspanins with the secretases not much is known about their physiological role, their importance in Alzheimer's Disease and their exact mode of action. This review aims to summarize the current knowledge and open questions regarding the biology of tetraspanins and the understanding how these proteins interact with APP processing pathways. Ultimately, it will be of interest if tetraspanins are suitable targets for future therapeutical approaches.

Keywords: tetraspanin, Alzheimer disease, membrane microdomains, amyloid precursor protein, secretases, amyloid beta

\section{INTRODUCTION}

The neurotoxic amyloid beta $(A \beta)$ peptide is a major component of senile plaques in Alzheimer's Disease $(\mathrm{AD})$ and derives from its precursor the amyloid precursor protein (APP). Despite an intensive effort and increasing understanding of its role in $\mathrm{AD}$ the physiological function of APP is not completely understood. APP and its relatives amyloid-like protein-1 (APLP1) and amyloid-like protein-2 (APLP2) are proteolytically processed, ubiquitously expressed and share overlapping functions. APP has been linked with trophic roles in neurons and synapses, axon pruning, intracellular signaling and apoptosis (Muller and Zheng, 2012). How APP interaction with other proteins is defined, how its proteolytic processing is controlled and how signaling events are regulated by APP is poorly understood. Proteomics-based approaches and yeast-two-hybrid screens have been used to identify the protein interaction network of APP 
(Kohli et al., 2012; Yu et al., 2015) and of the proteases known to cleave APP (Wakabayashi et al., 2009; Jeon et al., 2013). Among others, members of the tetraspanin family have been identified. Tetraspanins have been characterized as scaffold for protein interactions establishing tetraspanin-enriched microdomains (TEMs) and are involved in grouping APP and functional important protein partners.

This review focuses on the emerging role of tetraspanins in the regulation of the proteases involved in the proteolytic processing of APP. The available knowledge about how tetraspanins regulate processing and intracellular trafficking of APP and APP-cleaving secretases is summarized. It is discussed why tetraspanins are attractive novel drug targets. There are some excellent reviews covering different aspects of tetraspanin biology thereby providing a useful overview about their diverse functions (Berditchevski and Odintsova, 2007; Yanez-Mo et al., 2009; Charrin et al., 2014).

\section{WHAT ARE TETRASPANINS?}

Tetraspanins are compact and glycosylated transmembrane proteins, that span cell membranes four times. Two extracellular domains, one larger and one smaller loop are separated from three cytosolic domains, one short loop and one N-terminal and C-terminal end, respectively. Intracellular cysteine residues of the tetraspanins can be modified by lipidation, i.e., addition of palmitate, possibly contributing to the establishment of tetraspanin microdomains and the regulation of intracellular signaling events (Berditchevski et al., 2002; Charrin et al., 2002; Yang et al., 2002). The large extracellular loop (LEL) and the transmembrane domains play a role in mediating proteinprotein interactions (Hemler, 2003; Charrin et al., 2009). The structure of the isolated LEL of human CD81 was solved. It looks mushroom-shaped and it consists of a conserved subdomain, including three helices and a more variable one with two helices, possibly involved in the binding to other membrane proteins (Kitadokoro et al., 2001; Seigneuret et al., 2001). The full CD81 structure revealed a cone-like structure, where the LEL harbors an intramembrane cavity which is supposed to bind cholesterol (Zimmerman et al., 2016). It is speculated that the cholesterol bound structure favors a closed structural state of this tetraspanin with less tightly bound partner proteins.

Thirty three members of tetraspanins have been described. They can be mainly found at the plasma membrane and within endocytic membranes. Co-immunoprecipitation and crosslinking experiments revealed a high affinity of tetraspanins to interact with each other and other transmembrane proteins. These are in particular integrins, but also members of the immunoglobulin superfamily, signaling receptors, enzymes such as proteases and many other integral proteins residing in TEMs (Yanez-Mo et al., 2009).

\section{FUNCTIONS OF TETRASPANINS}

The function of tetraspanins is mainly defined by their ability to interact with other transmembrane proteins. Due to the great variety of partner proteins, tetraspanins are involved in various cellular processes like migration, adhesion, signaling and pathogen infection (Boucheix and Rubinstein, 2001; Lammerding et al., 2003; Barreiro et al., 2008). By regulating cell motility and different signaling pathways, tetraspanins play an important role in cancer progression and metastasis (Boucheix and Rubinstein, 2001; Wang et al., 2011). For example, the tetraspanins CD9 and CD151 contribute to cancer cell invasion by interacting with different integrins and signaling enzymes, like protein kinase $\mathrm{C}$ (PKC) and phosphoinositide 4-kinase (PI4K) (Zhang et al., 2001; Wang et al., 2011). Tetraspanins also modulate intracellular signaling pathways by coordinating ligand-receptor binding at the cell surface. This is exemplified by the observation that tetraspanin 3 promotes binding of the NogoA ligand to the receptor sphingosine-1-phosphate-receptor-2 (S1PR2), which activates an intracellular signaling cascade leading to the inhibition of neurite outgrowth (Thiede-Stan et al., 2015). Most tetraspanins regulate the functions of their partner proteins by modulating their spatiotemporal distribution at the plasma membrane and organizing them together with other functional proteins (e.g., enzymes and substrates) (Odintsova et al., 2003; Haining et al., 2012; Thiede-Stan et al., 2015). Recent studies, demonstrated that the interaction with tetraspanins influences the motility of their partner proteins and their association with other molecules within the plasma membrane (Yang et al., 2012; Mattila et al., 2013; Jouannet et al., 2015). In addition, some tetraspanins directly control the trafficking of their partner proteins (Berditchevski and Odintsova, 2007). For example CD63, facilitates endocytosis of the HIV receptor C-X-C chemokine receptor type 4 (Yoshida et al., 2008).

Further genetic and in vivo studies demonstrated the importance of tetraspanins in various physiological and pathophysiological processes. In the central nervous system, the knockout of CD81 increased brain size and number of glial cells in mice (Geisert et al., 2002). Tspan7 regulates spine maturation and AMPA receptor trafficking by interacting with the protein interacting with C-kinase 1 (PICK1) in rat hippocampal neurons (Bassani et al., 2012). Moreover, loss of CD9 in mice impaired formation of axoglial paranodal junctions and caused myelination deficits in the peripheral nervous system (Ishibashi et al., 2004). Also other tetraspanins like Tspan5 (Garcia-Frigola et al., 2001) and Tspan3 (Seipold et al., 2016) are highly expressed in the brain and in neuronal cells. However, their physiological roles remain unclear. Tetraspanin knockout mice additionally revealed the importance of CD9, CD81, CD37, and CD151 for fertilization, brain and peripheral nerve development and the immune response. However, analysis of tetraspanin functions by loss-of-function approaches in mice has been hampered, due to compensatory effects and their redundant functions.

In human, mutations of tetraspanin $7, \mathrm{CD} 151$ and the retinal tetraspanin Peripherin/RDS are associated with X-linked mental retardation, skin and kidney diseases, deafness and retinal degeneration (Kohl et al., 1998; Zemni et al., 2000; Karamatic Crew et al., 2004). 


\section{TETRASPANINS AS REGULATORS OF $\alpha$-SECRETASE ACTIVITY}

Several tetraspanins associate with the APP secretases and regulate their activity. In particular, the membrane localized $\alpha$ secretase ADAM10 associates with multiple tetraspanins. Using mild detergent conditions CD9, CD53, CD81, CD82, and CD151 were identified to associate with ADAM10. CD9, CD81, CD82 were able to stimulate ADAM10-dependent TNF $\alpha$ and EGF shedding (Arduise et al., 2008). In an independent study the association of ADAM10 with tetraspanin 12 (Tspan12) caused an accelerated ADAM10 maturation, i.e., the cleavage of the pro-ADAM10 to the mature and active protease, followed by an increased ADAM10-dependent APP processing (Xu et al., 2009). It was postulated that Tspan 12 activated proprotein convertases and stabilized the mature form of ADAM10. Coimmunoprecipitation experiments, performed under stringent detergent conditions, suggested that CD9, CD81, CD82, and CD151 did not directly interact with ADAM10 (Dornier et al., 2012). It was concluded that these tetraspanins associate with ADAM10 through interactions mediated by other members of the tetraspanin web. However, tetraspanins belonging to the TspanC8 subfamily still interacted with ADAM10 under stringent immunoprecipitation conditions, indicating that these tetraspanins directly bind to the protease (Dornier et al., 2012).

This evolutionary related subgroup of TspanC8 tetraspanins (Figure 1) includes the tetraspanins 5, 10, 14, 15, 17, and 33, which all contain eight conserved cysteine residues within their LEL. Analysis of the TspanC8-ADAM10 interaction revealed that overexpression of individual TspanC8 tetraspanins promoted ADAM10 maturation in human cells and Drosophila melanogaster (Haining et al., 2012). With exception of Tspan10 and Tspan17, TspanC8 overexpression also increased ADAM10 surface localization. Heterologous Tspan10 and Tspan17 expression led to a localization of ADAM10 to late endosomes (Dornier et al., 2012). Although, the C8 tetraspanins exert similar

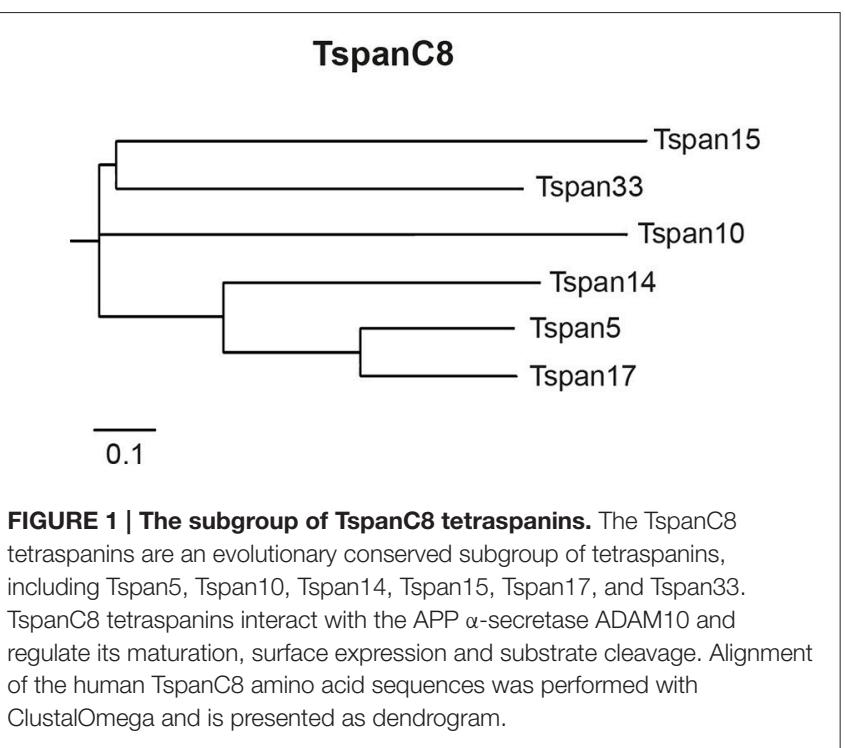

effects on ADAM10 maturation and trafficking (except Tspan10 and Tspan17), they have different impact on the cleavage of ADAM10 substrates (Prox et al., 2012; Noy et al., 2016). The overexpression of the TspanC8s members Tspan 5 and Tspan 14 promoted ligand induced shedding of the Notch receptor. In contrast, expression of Tspan15 reduced Notch processing (Dornier et al., 2012). Tspan15 was the only TspanC8 member, that increased ADAM10-mediated N-cadherin shedding after overexpression in human embryonic kidney (HEK293) and monkey fibroblast-like Cos7 cells (Prox et al., 2012; Noy et al., 2016). It was also shown that the generation of APP C-terminal fragments was differentially affected by certain TspanC8s. While expression of Tspan14 and Tspan33 slightly reduced the appearance of APP C-terminal cleavage products (Jouannet et al., 2015), Tspan5 expression had no effect on the production these fragments. Tspan 15 expression in the human osteosarcoma cell line U2OS-N1 (Jouannet et al., 2015) also reduced APP processing, but increased it in murine neuroblastoma (N2a) and HEK293 cells (Prox et al., 2012). Since, tetraspanins act in concert with other tetraspanins in TEMs the conflicting data may be explained by the different composition of tetraspanins in the different cellular systems used.

There is increasing evidence that TspanC8s mediate substrate specificity by a direct interaction with ADAM10 and modulation of its association with other membrane components, e.g., integrins (Jouannet et al., 2016). Using ADAM10 chimeric and truncation constructs, it was demonstrated that the TspanC8s differentially favor the interaction with the ADAM10 membrane proximal stalk region, cysteine-rich domain and disintegrin domain (Noy et al., 2016). TspanC8s may constrain and stabilize ADAM10 in defined conformations (Noy et al., 2016). The expression of TspanC8 tetraspanins had different impact on the membrane environment of ADAM10. Mass-spectrometric analysis of ADAM10-associated proteins revealed that in Tspan5 expressing cells ADAM10 preferably associated with classical components of the tetraspanin web such as the $\alpha 3 \beta 1$ integrin, CD9P1 and CD9, which was reduced in Tspan 15 transfected cells (Jouannet et al., 2015). Tspan5 expression enhanced ADAM10's localization at the cell periphery, while Tspan15 expression did not (Jouannet et al., 2015).

Tspan3, a TspanC6 tetraspanin, was identified, as another ADAM10 and APP interacting tetraspanin in cells and in the murine brain (Seipold et al., 2016). Tspan3 expression did not obviously influence ADAM10 maturation or trafficking but increased ADAM10-mediated APP cleavage. Tspan3 is likely involved in this process as a scaffold protein, which stabilizes ADAM10 and APP at the cell surface.

An interaction of tetraspanins with ADAM17, which is closely related to ADAM10 and under certain circumstances also exerts $\alpha$-secretase activity toward APP (Buxbaum et al., 1998), has only been described for CD9. Heterologous expression of CD9 or treatment with CD9-specific antibodies inhibited phorbol ester (PMA)-stimulated shedding of the ADAM17 substrates TNF $\alpha$ and ICAM-I, while CD9 knockdown increased it (Gutierrez-Lopez et al., 2011). In the same manner, treatment with neutralizing anti-CD9 monoclonal antibodies reduced ADAM17-mediated shedding of LR11 (SorLa) in human 
leukocytes, while CD9 expression increased it (Tsukamoto et al., 2014). Most likely CD9 inhibits ADAM17 sheddase activity by affecting the membrane compartmentalization of ADAM17 itself or its substrates. CD9 may even interact with both, ADAM10 and ADAM17, but exerting opposite effects on their activity with regards to TNF $\alpha$ shedding (Gutierrez-Lopez et al., 2011).

\section{TETRASPANINS AS REGULATORS OF $\gamma$-SECRETASE ACTIVITY}

Next to ADAM10 also the $\gamma$-secretase complex interacts with tetraspanins. Wakabayashi et al. showed that Presenilin-1 and Presenilin-2 associate with the tetraspanins CD9, CD81, Upk1b as well as with the tetraspanin associated proteins EWI-F, EWI-2 which connect the tetraspanin web with the actin cytoskeleton (Sala-Valdes et al., 2006) and CD98hc (Fenczik et al., 2001), a regulator of integrin signaling and amino acid transport (Wakabayashi et al., 2009). The activity of the $\gamma$-secretase complex was strongly decreased upon knockdown of CD81, EWI-F, and CD98hc, which correlated with a decrease in $A \beta$ production. Inhibition of $\gamma$-secretase activity was also observed in CD9 and CD81-deficient mouse embryonic fibroblasts revealed by an accumulation of C-terminal fragments of the $\gamma$-secretase substrates APP, APLP-2 ADAM10, N-Cadherin and Syndecan3. Treatment with anti-CD9 monoclonal antibodies reduced $A \beta$ levels in HEK293 cells (Wakabayashi et al., 2009). Additionally, siRNA mediated knockdown of Tspan33 in HeLa cells reduced the $\gamma$-secretase dependent cleavage of a constitutively active, truncated form of Notch1 and that of T-cell acute lymphoblastic leukemia (T-ALL) Notch1 oncogenic mutants (Dunn et al., 2010).

Independent studies analyzing the interactome of the $\gamma$ secretase complex identified the tetraspanins CD63 and Tspan3 in the network of the presenilin interacting proteins (Jeon et al., 2013; Seipold et al., 2016). CD63 is one of the few tetraspanins which is found on late endosomal and lysosomal membranes (Rous et al., 2002). CD63 associates with CD9, CD81, and CD82 within the tetraspanin web. However, the functional consequence of this interaction for the $\gamma$-secretase complex has not been elucidated. Due to its functions in the endosomal sorting complex required for transport (ESCRT)-independent formation of intraluminal vesicles (van Niel et al., 2011) CD63 could control the degradation of the $\gamma$-secretase complex.

\section{TETRASPANINS AS A PART OF A MULTI-SECRETASE COMPLEX}

Another mechanism by which tetraspanins regulate both, $\alpha$ - and $\gamma$-secretase, activity has recently been reported by Chen et al. (2015). It was shown that $\alpha$ - and $\gamma$-secretase associate in an active multiprotease complex at the plasma membrane (Figure 2). Assembly of this multi-secretase complex seems to be modified by Tspan 12 and the TspanC8 tetraspanins Tspan 5, 14, 12, and 17. Knockdown of Tspan5 and Tspan14 decreased ADAM10 association with the $\gamma$-secretase complex, which correlated with a reduced presence of mature ADAM10. Knockdown of Tspan 12 and Tspan17 also decreased the association of ADAM10 with the

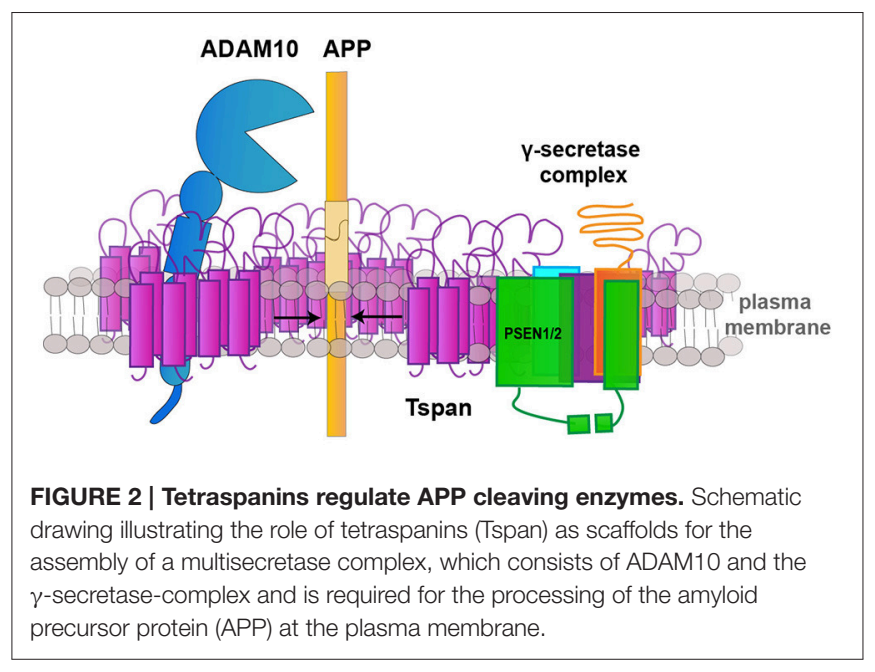

$\gamma$-secretase complex and the $\alpha$-secretase-dependent generation of soluble sAPP $\alpha$, but did not alter ADAM10 maturation. Moreover, Tspan12 and Tspan17 seem to contribute to an $\alpha-/ \gamma$-secretase feedback mechanism. This feedback mechanism is related to $\gamma$-secretase inhibition and causes an increase of $\operatorname{sAPP} \alpha$ at the expense of sAPP $\beta$. This is also accompanied by an increase of APP and BACE1 surface levels. This effect was less effective after knockdown of Tspan12 and Tspan17 (Chen et al., 2015).

In conclusion, tetraspanins are potent regulators of $\alpha$ and $\gamma$-secretase activity, which modulate maturation, complex assembly, trafficking and substrate specificity. In regards to $\beta$ secretase cleavage, no direct interaction of tetraspanins with the $\beta$-secretase BACE1 has been reported. The metalloprotease Meprin $\beta$, which cleaves APP in a manner similar to BACE1 (Bien et al., 2012), interacts with Tspan 8 and resides together with APP in TEMs (Schmidt et al., 2016). However, Tspan8 had no impact on the proteolytic activity of Meprin $\beta$ towards APP.

\section{TETRASPANINS AS THERAPEUTIC TARGETS}

The treatment with monoclonal antibodies (mAbs) against CD81 diminished the development of neurological symptoms in a multiple sclerosis mouse model (Dijkstra et al., 2008) and prevented hepatitis $\mathrm{C}$ virus $(\mathrm{HCV})$ infection after prophylactic injection in mice (Meuleman et al., 2008). Application of anti-CD9 antibodies reduced tumor growth and progression in gastric cancer mouse xenografts (Nakamoto et al., 2009). Stimulatory CD151 antibodies promoted cell adhesion and thereby reduced immobilization of tumor cells and metastasis (Zijlstra et al., 2008). The humanized anti-CD37 IgG fusion protein Otlertuzumab (TRU-016) is a potential drug for the treatment of lymphoid B-cell malignancies (Robak et al., 2009) and was tested in phase 1 clinical trials for the treatment of chronic lymphocytic leukemia (Byrd et al., 2014). It is proposed that $\mathrm{mAbs}$ directed against tetraspanins inhibit lateral associations or cause the formation of tetraspanin aggregates, which disrupt TEMs and cause a downregulation of the targeted 
tetraspanin and its partner protein(s) (Hemler, 2008). Also recombinant soluble large extracellular domains (sLEL) may inhibit tetraspanin-dependent functions. Similar to mAbs, CD81 sLELs reduced HCV infectivity and blocked HIV-1 entry into macrophages (Flint et al., 2006; Ho et al., 2006).

The potential of tetraspanins to modulate $\gamma$-secretase activity in $\mathrm{AD}$ was demonstrated by RNAi-mediated knockdown experiments. The downregulation of CD81 and tetraspaninassociated proteins EWI-F and CD98hc reduced the secretion of neurotoxic $A \beta$ in HEK cells, stably overexpressing a mutated form of APP, which favors amyloidogenic processing. Likewise, treatment with anti-CD81 and anti-CD9 mAbs decreased A $\beta$ production in HEK293 cells (Wakabayashi et al., 2009). However, most therapeutic approaches targeting $\gamma$-secretase activity were accompanied by severe side effects, like skin cancer development, gastrointestinal toxicity and infections (Doody et al., 2013), due to the physiological role of its substrates, for example Notch1 (Haapasalo and Kovacs, 2011). Interestingly, the individual knockdown of CD9 and CD81 in HeLa cells had no significant effect on the activity of different leukemic mutant forms of Notch1 (Dunn et al., 2010). It was further shown that human Tspan33 promotes $\gamma$-secretase cleavage of Notch and that depletion of Tspan33 might be a potential target in T-ALL, a rare yet aggressive form of lymphoblastic leukemia, which is associated with activating mutations of Notch1 (Weng et al., 2004; Dunn et al., 2010). Since CD9, CD81, and Tspan33 are also regarded as regulators of ADAM10- and ADAM17 (Arduise et al., 2008; Gutierrez-Lopez et al., 2011; Haining et al., 2012; Jouannet et al., 2016), the effects of potential therapeutics have to be studied carefully.

By reducing $A \beta$ - and increasing sAPP $\alpha$-production the upregulation of ADAM10 expression had beneficial effects in an AD mouse model (Postina et al., 2004). ADAM10 is another promising target for the treatment of $\mathrm{AD}$, as demonstrated by a recent study using the synthetic retinoid acitretin to increase ADAM10 expression in $\mathrm{AD}$ patients (Endres et al., 2014). Targeting specific members of the TspanC8s, which enhance ADAM10 activity, but have different impact on its substrate specificity, could possibly reduce side effects of a global ADAM10 activation. Moreover, most of the TspanC8s are not expressed in all cell-types (Dornier et al., 2012; Jouannet et al., 2016), indicating that targeting these tetraspanins could regulate ADAM10 activity in a cell-type specific manner. With regard to $\mathrm{AD}$, enhancing ADAM10 non-amyloidogenic APP processing could be achieved by stimulation of Tspan12, Tspan 15 and Tspan33 using agonistic monoclonal antibodies, sLELs or small molecular drugs that increase the promoter activity and protein expression of these tetraspanins.

ADAM10 is also associated with tumor progression, metastasis and inflammation by site-specific cleavage of several adhesion molecules and cytokines. In this case a downregulation of its proteolytic activity could be of therapeutic benefit (Moss et al., 2008; Saftig and Reiss, 2011). ADAM10-mediated $\mathrm{N}$-cadherin shedding was associated with cancer cell migration
(Kohutek et al., 2009) promoting tumor progression and metastasis. In this regard downregulation of Tspan5 and Tspan15, which predominantly promote ADAM10-mediated $\mathrm{N}$-cadherin shedding (Noy et al., 2016), by antagonistic mAbs, sLELs or RNAi, could be a therapeutic option. By sharing several substrates with ADAM10, inhibition of ADAM17 is also effective in different kinds of cancer and inflammatory disorders (Saftig and Reiss, 2011). The expression of CD9 reduced ADAM17dependent TNF $\alpha$ shedding (Gutierrez-Lopez et al., 2011), which is a main factor in inflammation and involved in rheumatoid arthritis, psoriasis and inflammatory bowel disease.

To evaluate the full therapeutic potential of tetraspanins, the exact mechanisms and consequences of potential tetraspanindirected therapeutics need to be further investigated. Due to their multiple interaction partners and the complex organization in TEMs, tetraspanins also have opposing functions, which might depend on the cellular system. While downregulation of Tspan33 in HeLa cells decreased Notch1 signaling (Dunn et al., 2010), its overexpression in U2OS-N1 cells reduced Notch1 activity (Jouannet et al., 2016). Targeting such tetraspanins could cause severe adverse effects such as cancer development and inflammation. Moreover, redundancy of tetraspanin functions and compensatory effects might decrease the clinical activity of potential therapeutics.

\section{CONCLUSION}

In summary, tetraspanins are potent regulators of APP cleaving enzymes. In particular, tetraspanins came into focus as cell-type and substrate specific regulators of the $\alpha$-secretases ADAM10 but also of the $\gamma$-secretase complex.

Their specific functions and localization make tetraspanins an interesting target for the treatment of $\mathrm{AD}$ and possibly other diseases. However, first approaches trying to target tetraspanins have not succeeded, which could be explained by their functional redundancy. It will be necessary to better understand how tetraspanins exactly work and how their redundancy is regulated. Another central aspect is how tetraspanin expression is regulated and if tetraspanin dysfunctions are associated with the development of AD.

\section{AUTHOR CONTRIBUTIONS}

Both authors wrote the review manuscript. LS designed the figure art.

\section{FUNDING}

Work in the laboratory of PS has been funded through grants of the Deutsche Forschungsgemeinschaft (DFG) in the SFB877, A3, and A12; through support of the VERUM foundation, the Alzheimer Research Price of the Breuer Foundation and the Interuniversity Attraction Poles Program IUAP P7/16 of the Belgian Federal Science Policy Office. 


\section{REFERENCES}

Arduise, C., Abache, T., Li, L., Billard, M., Chabanon, A., Ludwig, A., et al. (2008). Tetraspanins regulate ADAM10-mediated cleavage of TNF- $\alpha$ and epidermal growth factor. J. Immunol. 181, 7002-7013. doi: 10.4049/jimmunol.181.10.7002

Barreiro, O., Zamai, M., Yanez-Mo, M., Tejera, E., Lopez-Romero, P., Monk, P. N., et al. (2008). Endothelial adhesion receptors are recruited to adherent leukocytes by inclusion in preformed tetraspanin nanoplatforms. J. Cell Biol. 183, 527-542. doi: 10.1083/jcb.200805076

Bassani, S., Cingolani, L. A., Valnegri, P., Folci, A., Zapata, J., Gianfelice, A., et al. (2012). The X-linked intellectual disability protein TSPAN7 regulates excitatory synapse development and AMPAR trafficking. Neuron 73, 1143-1158. doi: 10.1016/j.neuron.2012.01.021

Berditchevski, F., and Odintsova, E. (2007). Tetraspanins as regulators of protein trafficking. Traffic 8, 89-96. doi: 10.1111/j.1600-0854.2006.00515.x

Berditchevski, F., Odintsova, E., Sawada, S., and Gilbert, E. (2002). Expression of the palmitoylation-deficient CD151 weakens the association of $\alpha_{3} \beta_{1}$ integrin with the tetraspanin-enriched microdomains and affects integrin-dependent signaling. J. Biol. Chem. 277, 36991-37000. doi: 10.1074/jbc.M205265200

Bien, J., Jefferson, T., Causevic, M., Jumpertz, T., Munter, L., Multhaup, G., et al. (2012). The metalloprotease meprin $\beta$ generates amino terminaltruncated amyloid $\beta$ peptide species. J. Biol. Chem. 287, 33304-33313. doi: 10.1074/jbc.M112.395608

Boucheix, C., and Rubinstein, E. (2001). Tetraspanins. Cell. Mol. Life Sci. 58, 1189-1205. doi: 10.1007/PL00000933

Buxbaum, J. D., Liu, K. N., Luo, Y., Slack, J. L., Stocking, K. L., Peschon, J. J., et al. (1998). Evidence that tumor necrosis factor $\alpha$ converting enzyme is involved in regulated $\alpha$-secretase cleavage of the Alzheimer amyloid protein precursor. J. Biol. Chem. 273, 27765-27767. doi: 10.1074/jbc.273.43.27765

Byrd, J. C., Pagel, J. M., Awan, F. T., Forero, A., Flinn, I. W., DeaunaLimayo, D. P., et al. (2014). A phase 1 study evaluating the safety and tolerability of otlertuzumab, an anti-CD37 mono-specific ADAPTIR therapeutic protein in chronic lymphocytic leukemia. Blood 123, 1302-1308. doi: 10.1182/blood-2013-07-512137

Charrin, S., Jouannet, S., Boucheix, C., and Rubinstein, E. (2014). Tetraspanins at a glance. J. Cell Sci. 127, 3641-3648. doi: 10.1242/jcs. 154906

Charrin, S., le Naour, F., Silvie, O., Milhiet, P. E., Boucheix, C., and Rubinstein, E. (2009). Lateral organization of membrane proteins: tetraspanins spin their web. Biochem. J. 420, 133-154. doi: 10.1042/BJ20082422

Charrin, S., Manie, S., Oualid, M., Billard, M., Boucheix, C., and Rubinstein, E. (2002). Differential stability of tetraspanin/tetraspanin interactions: role of palmitoylation. FEBS Lett. 516, 139-144. doi: 10.1016/S0014-5793(02)02522-X

Chen, A. C., Kim, S., Shepardson, N., Patel, S., Hong, S., and Selkoe, D. J. (2015). Physical and functional interaction between the $\alpha$ - and $\gamma$-secretases: a new model of regulated intramembrane proteolysis. J. Cell Biol. 211, 1157-1176. doi: $10.1083 /$ jcb. 201502001

Dijkstra, S., Kooij, G., Verbeek, R., van der Pol, S. M., Amor, S., Geisert, E. E. Jr., et al. (2008). Targeting the tetraspanin CD81 blocks monocyte transmigration and ameliorates EAE. Neurobiol. Dis. 31, 413-421. doi: 10.1016/j.nbd.2008.05.018

Doody, R. S., Raman, R., Farlow, M., Iwatsubo, T., Vellas, B., Joffe, S., et al. (2013). A phase 3 trial of semagacestat for treatment of Alzheimer's disease. N. Engl. J. Med. 369, 341-350. doi: 10.1056/NEJMoa1210951

Dornier, E., Coumailleau, F., Ottavi, J. F., Moretti, J., Boucheix, C., Mauduit, P., et al. (2012). TspanC8 tetraspanins regulate ADAM10/Kuzbanian trafficking and promote Notch activation in flies and mammals. J. Cell Biol. 199, 481-496. doi: $10.1083 /$ jcb.201201133

Dunn, C. D., Sulis, M. L., Ferrando, A. A., and Greenwald, I. (2010). A conserved tetraspanin subfamily promotes Notch signaling in Caenorhabditis elegans and in human cells. Proc. Natl. Acad. Sci. U.S.A. 107, 5907-5912. doi: 10.1073/pnas.1001647107

Endres, K., Fahrenholz, F., Lotz, J., Hiemke, C., Teipel, S., Lieb, K., et al. (2014). Increased CSF APPs- $\alpha$ levels in patients with Alzheimer disease treated with acitretin. Neurology 83, 1930-1935. doi: 10.1212/WNL.0000000000001017

Fenczik, C. A., Zent, R., Dellos, M., Calderwood, D. A., Satriano, J., Kelly, C., et al. (2001). Distinct domains of CD98hc regulate integrins and amino acid transport. J. Biol. Chem. 276, 8746-8752. doi: 10.1074/jbc.M011239200
Flint, M., von Hahn, T., Zhang, J., Farquhar, M., Jones, C. T., Balfe, P., et al. (2006). Diverse CD81 proteins support hepatitis C virus infection. J. Virol. 80, 11331-11342. doi: 10.1128/JVI.00104-06

Garcia-Frigola, C., Burgaya, F., de Lecea, L., and Soriano, E. (2001). Pattern of expression of the tetraspanin Tspan-5 during brain development in the mouse. Mech. Dev. 106, 207-212. doi: 10.1016/S0925-4773(01)00436-1

Geisert, E. E. Jr., Williams, R. W., Geisert, G. R., Fan, L., Asbury, A. M., Maecker, H. T., et al. (2002). Increased brain size and glial cell number in CD81-null mice. J. Comp. Neurol. 453, 22-32. doi: 10.1002/cne.10364

Gutierrez-Lopez, M. D., Gilsanz, A., Yanez-Mo, M., Ovalle, S., Lafuente, E. M., Dominguez, C., et al. (2011). The sheddase activity of ADAM17/TACE is regulated by the tetraspanin CD9. Cell. Mol. Life Sci. 68, 3275-3292. doi: 10.1007/s00018-011-0639-0

Haapasalo, A., and Kovacs, D. M. (2011). The many substrates of presenilin/gamma-secretase. J. Alzheimers. Dis. 25, 3-28. doi: 10.3233/ JAD-2011-101065

Haining, E. J., Yang, J., Bailey, R. L., Khan, K., Collier, R., Tsai, S., et al. (2012). The TspanC8 subgroup of tetraspanins interacts with A disintegrin and metalloprotease 10 (ADAM10) and regulates its maturation and cell surface expression. J. Biol. Chem. 287, 39753-39765. doi: 10.1074/jbc.M112.416503

Hemler, M. E. (2003). Tetraspanin proteins mediate cellular penetration, invasion, and fusion events and define a novel type of membrane microdomain. Annu. Rev. Cell Dev. Biol. 19, 397-422. doi: 10.1146/annurev.cellbio.19.111301.153609

Hemler, M. E. (2008). Targeting of tetraspanin proteins-potential benefits and strategies. Nat. Rev. Drug Discov. 7, 747-758. doi: 10.1038/nrd2659

Ho, S. H., Martin, F., Higginbottom, A., Partridge, L. J., Parthasarathy, V., Moseley, G. W., et al. (2006). Recombinant extracellular domains of tetraspanin proteins are potent inhibitors of the infection of macrophages by human immunodeficiency virus type 1. J. Virol. 80, 6487-6496. doi: 10.1128/JVI.02539-05

Ishibashi, T., Ding, L., Ikenaka, K., Inoue, Y., Miyado, K., Mekada, E., et al. (2004). Tetraspanin protein CD9 is a novel paranodal component regulating paranodal junctional formation. J. Neurosci. 24, 96-102. doi: 10.1523/JNEUROSCI.1484-03.2004

Jeon, A. H., Bohm, C., Chen, F., Huo, H., Ruan, X., Ren, C. H., et al. (2013). Interactome analyses of mature gamma-secretase complexes reveal distinct molecular environments of presenilin (PS) paralogs and preferential binding of signal peptide peptidase to PS2. J. Biol. Chem. 288, 15352-15366. doi: 10.1074/jbc.M112.441840

Jouannet, S., Saint-Pol, J., Fernandez, L., Nguyen, V., Charrin, S., Boucheix, C., et al. (2015). TspanC8 tetraspanins differentially regulate the cleavage of ADAM10 substrates, Notch activation and ADAM10 membrane compartmentalization. Cell. Mol. Life Sci. 73, 1895-1915. doi: 10.1007/s00018-015-2111-z

Jouannet, S., Saint-Pol, J., Fernandez, L., Nguyen, V., Charrin, S., Boucheix, C., et al. (2016). TspanC8 tetraspanins differentially regulate the cleavage of ADAM10 substrates, Notch activation and ADAM10 membrane compartmentalization. Cell. Mol. Life Sci. 73, 1895-1915. doi: 10.1007/s00018-015-2111-z

Karamatic Crew, V., Burton, N., Kagan, A., Green, C. A., Levene, C., Flinter, F., et al. (2004). CD151, the first member of the tetraspanin (TM4) superfamily detected on erythrocytes, is essential for the correct assembly of human basement membranes in kidney and skin. Blood 104, 2217-2223. doi: 10.1182/blood-2004-04-1512

Kitadokoro, K., Bordo, D., Galli, G., Petracca, R., Falugi, F., Abrignani, S., et al. (2001). CD81 extracellular domain 3D structure: insight into the tetraspanin superfamily structural motifs. EMBO J. 20, 12-18. doi: 10.1093/emboj/20.1.12

Kohl, S., Giddings, I., Besch, D., Apfelstedt-Sylla, E., Zrenner, E., and Wissinger, B. (1998). The role of the peripherin/RDS gene in retinal dystrophies. Acta Anat. (Basel). 162, 75-84. doi: 10.1159/000046471

Kohli, B. M., Pflieger, D., Mueller, L. N., Carbonetti, G., Aebersold, R., Nitsch, R. M., et al. (2012). Interactome of the amyloid precursor protein APP in brain reveals a protein network involved in synaptic vesicle turnover and a close association with Synaptotagmin-1. J. Proteome Res. 11, 4075-4090. doi: 10.1021/pr300123g

Kohutek, Z. A., diPierro, C. G., Redpath, G. T., and Hussaini, I. M. (2009). ADAM-10-mediated N-cadherin cleavage is protein kinase $\mathrm{C}-\alpha$ dependent 
and promotes glioblastoma cell migration. J. Neurosci. 29, 4605-4615. doi: 10.1523/JNEUROSCI.5126-08.2009

Lammerding, J., Kazarov, A. R., Huang, H., Lee, R. T., and Hemler, M. E. (2003). Tetraspanin CD151 regulates $\alpha_{6} \beta_{1}$ integrin adhesion strengthening. Proc. Natl. Acad. Sci. U.S.A. 100, 7616-7621. doi: 10.1073/pnas.1337546100

Mattila, P. K., Feest, C., Depoil, D., Treanor, B., Montaner, B., Otipoby, K. L., et al. (2013). The actin and tetraspanin networks organize receptor nanoclusters to regulate B cell receptor-mediated signaling. Immunity 38, 461-474. doi: 10.1016/j.immuni.2012.11.019

Meuleman, P., Hesselgesser, J., Paulson, M., Vanwolleghem, T., Desombere, I., Reiser, H., et al. (2008). Anti-CD81 antibodies can prevent a hepatitis C virus infection in vivo. Hepatology 48, 1761-1768. doi: 10.1002/hep.22547

Moss, M. L., Stoeck, A., Yan, W., and Dempsey, P. J. (2008). ADAM10 as a target for anti-cancer therapy. Curr. Pharm. Biotechnol. 9, 2-8. doi: $10.2174 / 138920108783497613$

Muller, U. C., and Zheng, H. (2012). Physiological functions of APP family proteins. Cold Spring Harb. Perspect. Med. 2:a006288. doi: $10.1101 /$ cshperspect.a006288

Nakamoto, T., Murayama, Y., Oritani, K., Boucheix, C., Rubinstein, E., Nishida, M., et al. (2009). A novel therapeutic strategy with anti-CD9 antibody in gastric cancers. J. Gastroenterol. 44, 889-896. doi: 10.1007/s00535-009-0081-3

Noy, P. J., Yang, J., Reyat, J. S., Matthews, A. L., Charlton, A. E., Furmston, J., et al. (2016). TspanC8 tetraspanins and a disintegrin and metalloprotease 10 (ADAM10) interact via their extracellular regions: evidence for distinct binding mechanisms for different TspanC8 proteins. J. Biol. Chem. 291, 3145-3157. doi: 10.1074/jbc.M115.703058

Odintsova, E., Voortman, J., Gilbert, E., and Berditchevski, F. (2003). Tetraspanin CD82 regulates compartmentalisation and ligand-induced dimerization of EGFR. J. Cell Sci. 116, 4557-4566. doi: 10.1242/jcs.00793

Postina, R., Schroeder, A., Dewachter, I., Bohl, J., Schmitt, U., Kojro, E., et al. (2004). A disintegrin-metalloproteinase prevents amyloid plaque formation and hippocampal defects in an Alzheimer disease mouse model. J. Clin. Invest. 113, 1456-1464. doi: 10.1172/JCI20864

Prox, J., Willenbrock, M., Weber, S., Lehmann, T., Schmidt-Arras, D., Schwanbeck, R., et al. (2012). Tetraspanin 15 regulates cellular trafficking and activity of the ectodomain sheddase ADAM10. Cell. Mol. Life Sci. 69, 2919-2932. doi: 10.1007/s00018-012-0960-2

Robak, T., Robak, P., and Smolewski, P. (2009). TRU-016, a humanized anti-CD37 IgG fusion protein for the potential treatment of B-cell malignancies. Curr. Opin. Investig. Drugs 10, 1383-1390.

Rous, B. A., Reaves, B. J., Ihrke, G., Briggs, J. A., Gray, S. R., Stephens, D. J., et al. (2002). Role of adaptor complex AP-3 in targeting wild-type and mutated CD63 to lysosomes. Mol. Biol. Cell 13, 1071-1082. doi: 10.1091/mbc.01-08-0409

Saftig, P., and Reiss, K. (2011). The "A Disintegrin And Metalloproteases" ADAM10 and ADAM17: novel drug targets with therapeutic potential? Eur. J. Cell Biol. 90, 527-535. doi: 10.1016/j.ejcb.2010.11.005

Sala-Valdes, M., Ursa, A., Charrin, S., Rubinstein, E., Hemler, M. E., SanchezMadrid, F., et al. (2006). EWI-2 and EWI-F link the tetraspanin web to the actin cytoskeleton through their direct association with ezrin-radixinmoesin proteins. J. Biol. Chem. 281, 19665-19675. doi: 10.1074/jbc.M602 116200

Schmidt, F., Müller, M., Prox, J., Arnold, P., Schönherr, C., Tredup, C., et al. (2016). Tetraspanin 8 is an interactor of the metalloprotease meprin $\beta$ within tetraspanin-enriched microdomains. Biol. Chem. 397, 857-869. doi: 10.1515/hsz-2016-0126

Seigneuret, M., Delaguillaumie, A., Lagaudriere-Gesbert, C., and Conjeaud, H. (2001). Structure of the tetraspanin main extracellular domain. A partially conserved fold with a structurally variable domain insertion. J. Biol. Chem. 276, 40055-40064. doi: 10.1074/jbc.M105557200

Seipold, L., Damme, M., Prox, J., Rabe, B., Kasparek, P., Sedlacek, R., et al. (2016). Tetraspanin 3: a central endocytic membrane component regulating the expression of ADAM10, presenilin and the amyloid precursor protein. Biochim. Biophys. Acta. 1864, 217-230. doi: 10.1016/j.bbamcr.2016.11.003

Thiede-Stan, N. K., Tews, B., Albrecht, D., Ristic, Z., Ewers, H., and Schwab, M. E. (2015). Tetraspanin-3 is an organizer of the multi-subunit Nogo-A signaling complex. J. Cell Sci. 128, 3583-3596. doi: 10.1242/jcs.167981
Tsukamoto, S., Takeuchi, M., Kawaguchi, T., Togasaki, E., Yamazaki, A., Sugita, Y., et al. (2014). Tetraspanin CD9 modulates ADAM17-mediated shedding of LR11 in leukocytes. Exp. Mol. Med. 46:e89. doi: 10.1038/emm. 2013.161

van Niel, G., Charrin, S., Simoes, S., Romao, M., Rochin, L., Saftig, P., et al. (2011). The tetraspanin CD63 regulates ESCRT-independent and dependent endosomal sorting during melanogenesis. Dev. Cell 21, 708-721. doi: 10.1016/j.devcel.2011.08.019

Wakabayashi, T., Craessaerts, K., Bammens, L., Bentahir, M., Borgions, F., Herdewijn, P., et al. (2009). Analysis of the gamma-secretase interactome and validation of its association with tetraspanin-enriched microdomains. Nat. Cell Biol. 11, 1340-1346. doi: 10.1038/ncb1978

Wang, H. X., Li, Q., Sharma, C., Knoblich, K., and Hemler, M. E. (2011). Tetraspanin protein contributions to cancer. Biochem. Soc. Trans. 39, 547-552. doi: 10.1042/BST0390547

Weng, A. P., Ferrando, A. A., Lee, W., Morris, J. P. T., Silverman, L. B., Sanchez-Irizarry, C., et al. (2004). Activating mutations of NOTCH1 in human $\mathrm{T}$ cell acute lymphoblastic leukemia. Science 306, 269-271. doi: $10.1126 /$ science. 1102160

Xu, D., Sharma, C., and Hemler, M. E. (2009). Tetraspanin 12 regulates ADAM10dependent cleavage of amyloid precursor protein. FASEB J. 23, 3674-3681. doi: 10.1096/fj.09-133462

Yanez-Mo, M., Barreiro, O., Gordon-Alonso, M., Sala-Valdes, M., and Sanchez-Madrid, F. (2009). Tetraspanin-enriched microdomains: a functional unit in cell plasma membranes. Trends Cell Biol. 19, 434-446. doi: $10.1016 /$ j.tcb.2009.06.004

Yang, X., Claas, C., Kraeft, S. K., Chen, L. B., Wang, Z., Kreidberg, J. A., et al. (2002). Palmitoylation of tetraspanin proteins: modulation of CD151 lateral interactions, subcellular distribution, and integrin-dependent cell morphology. Mol. Biol. Cell 13, 767-781. doi: 10.1091/mbc.01-05-0275

Yang, X. H., Mirchev, R., Deng, X., Yacono, P., Yang, H. L., Golan, D. E., et al. (2012). CD151 restricts the $\alpha 6$ integrin diffusion mode. J. Cell Sci. 125, 1478-1487. doi: 10.1242/jcs.093963

Yoshida, T., Kawano, Y., Sato, K., Ando, Y., Aoki, J., Miura, Y., et al. (2008). A CD63 mutant inhibits T-cell tropic human immunodeficiency virus type 1 entry by disrupting CXCR4 trafficking to the plasma membrane. Traffic 9, 540-558. doi: 10.1111/j.1600-0854.2007.00700.x

Yu, Y., Li, Y., and Zhang, Y. (2015). Screening of APP interaction proteins by DUALmembrane yeast two-hybrid system. Int. J. Clin. Exp. Pathol. 8, 2802-2808.

Zemni, R., Bienvenu, T., Vinet, M. C., Sefiani, A., Carrie, A., Billuart, P., et al. (2000). A new gene involved in X-linked mental retardation identified by analysis of an X;2 balanced translocation. Nat. Genet. 24, 167-170. doi: $10.1038 / 72829$

Zhang, X. A., Bontrager, A. L., and Hemler, M. E. (2001). Transmembrane4 superfamily proteins associate with activated protein kinase $\mathrm{C}$ (PKC) and link PKC to specific $\beta_{1}$ integrins. J. Biol. Chem. 276, 25005-25013. doi: 10.1074/jbc.M102156200

Zijlstra, A., Lewis, J., Degryse, B., Stuhlmann, H., and Quigley, J. P. (2008). The inhibition of tumor cell intravasation and subsequent metastasis via regulation of in vivo tumor cell motility by the tetraspanin CD151. Cancer Cell 13, 221-234. doi: 10.1016/j.ccr.2008.01.031

Zimmerman, B., Kelly, B., McMillan, B. J., Seegar, T. C., Dror, R. O., Kruse, A. C., et al. (2016). Crystal structure of a full-length human tetraspanin reveals a cholesetrol-binding pocket. Cell 167, 1071-1051. doi: 10.1016/j.cell.2016.09.056

Conflict of Interest Statement: The authors declare that the research was conducted in the absence of any commercial or financial relationships that could be construed as a potential conflict of interest.

Copyright (c) 2016 Seipold and Saftig. This is an open-access article distributed under the terms of the Creative Commons Attribution License (CC BY). The use, distribution or reproduction in other forums is permitted, provided the original author(s) or licensor are credited and that the original publication in this journal is cited, in accordance with accepted academic practice. No use, distribution or reproduction is permitted which does not comply with these terms. 